\title{
Intratumoral Wnt2B expression affects tumor proliferation and survival in malignant pleural mesothelioma patients
}

\author{
MASASHI KOBAYASHI ${ }^{1}$, CHENG-LONG HUANG ${ }^{1}$, MAKOTO SONOBE $^{1}$, RYUTARO KIKUCHI ${ }^{1}$, \\ MASASHI ISHIKAWA ${ }^{1}$, JIRO KITAMURA ${ }^{1}$, RYO MIYAHARA ${ }^{1}$, TOSHI MENJU ${ }^{1}$, SHOTARO IWAKIRI ${ }^{2}$, \\ KAZUMI ITOI $^{2}$, RYOJI YASUMIZU ${ }^{3}$ and HIROSHI DATE ${ }^{1}$ \\ ${ }^{1}$ Department of Thoracic Surgery, Faculty of Medicine, Kyoto University, Shogoin, Sakyo-ku, Kyoto; \\ ${ }^{2}$ Department of Thoracic Surgery, Hyogo Prefectural Amagasaki Hospital, Amagasaki; \\ ${ }^{3}$ Department of Pathology, Hyogo Prefectural Tsukaguchi Hospital, Amagasaki, Japan
}

Received January 11, 2012; Accepted February 20, 2012

DOI: 10.3892/etm.2012.511

\begin{abstract}
Malignant pleural mesothelioma (MPM) is an aggressive thoracic tumor with a poor prognosis. We performed a comprehensive clinical study on the intratumoral expression of Wnt1, Wnt2B and Wnt5A in MPM. One hundred and seven MPM patients were investigated. Immunohistochemistry was performed to evaluate the intratumoral expression of Wnt1, Wnt2B, Wnt5A, survivin and c-Myc, and the Ki-67 proliferation index. The apoptotic index was evaluated by the TUNEL method. Among the 107 MPMs, 23 MPMs (21.5\%) were Wnt1-high tumors, 72 MPMs (67.3\%) were Wnt2B-high tumors and 54 MPMs $(50.5 \%)$ were Wnt5A-high tumors. There was no correlation among the levels of Wnt expression. The percentage of Wnt2B-positive tumors was significantly higher compared to that of the other Wnts $(\mathrm{p}<0.0001)$. Furthermore, intratumoral Wnt2B expression significantly correlated with the expression of survivin $(\mathrm{p}<0.001)$ and $\mathrm{c}-\mathrm{Myc}(\mathrm{p}<0.001)$. Regarding tumor biology, the Ki-67 proliferation index was significantly higher in the Wnt2B-high tumors than in the Wnt2B-low tumors $(\mathrm{p}=0.0438)$. In addition, the overall survival was significantly lower in patients with Wnt2B-high tumors than in those with Wnt2B-low tumors $(\mathrm{p}=0.0238)$. A Cox multivariate analysis also demonstrated the Wnt2B status to be a significant prognostic factor in MPM patients ( $p=0.0042$ ). Intratumoral Wnt2B expression was associated with the expression of survivin and c-Myc, tumor proliferation and patient survival in MPM. Wnt2B is a potential molecular target for the treatment of Wnt2B-overexpressing MPMs.
\end{abstract}

Correspondence to: Dr Cheng-Long Huang, Department of Thoracic Surgery, Faculty of Medicine, Kyoto University, 54 Kawahara-cho, Shogoin, Sakyo-ku, Kyoto 606-8507, Japan

E-mail: chuang@kuhp.kyoto-u.ac.jp

Key words: Wnt2B, survivin, c-Myc, proliferation, prognosis, mesothelioma

\section{Introduction}

Malignant pleural mesothelioma (MPM) is a thoracic tumor that arises from surface serosal cells of the pleura. It has been reported to be associated with inhalation exposure to asbestos (1). MPM is characterized by rapidly progressive and diffusely local growth, and a poor prognosis. Unfortunately, the incidence of MPM has been predicted to steadily increase and peak over the next two decades (2). However, there is no known curative modality for MPM (3), and long term survival is rare even with aggressive multimodal therapy including extrapleural pneumonectomy (4). Therefore, a new treatment strategy is required for MPM patients.

Among various molecules, the Wnt gene family encodes multi-functional signaling glycoproteins that are involved in the regulation of a wide variety of normal and pathological processes, including embryogenesis and tumorigenesis $(5,6)$. Recently, we found that three members of the Wnt family, including Wnt1, Wnt2B and Wnt5A, are associated with tumorigenesis (7). In fact, many clinical studies have reported that overexpression of Wnt members is associated with various tumorigenic processes, such as proliferation, angiogenesis and patient survival (8-11). However, there are only a few clinical studies on the molecular biology of human MPM (12).

Therefore, to clarify the tumor biology of MPM, we performed a comprehensive clinical study on the intratumoral expression of Wnt1, Wnt2B and Wnt5A, in relation to the tumor-associated Wnt targets, survivin $(13,14)$ and c-Myc $(15)$.

\section{Materials and methods}

Clinical characteristics of patients. One hundred and seven consecutive MPM patients who were diagnosed at Kyoto University Hospital, Hyogo Prefectural Amagasaki Hospital, or Hyogo Prefectural Tsukaguchi Hospital, from January 1998 to December 2010 were studied. This study was approved by the Ethics Committee of Kyoto University, and informed consent was obtained from each patient. All tumors were clinically staged according to the IMIG staging system (16), and histological classification was based on the WHO classification (17). The clinical records and histopathological diagnosis 
of all patients were fully documented. This report includes the follow-up data up to December 28, 2010.

Immunohistochemistry. The following antibodies were used: a rabbit polyclonal antibody for Wnt1 (H89; Santa Cruz Biotechnology, Inc., Santa Cruz, CA, USA) diluted at 1:200, a rabbit polyclonal antibody for Wnt2B (LS-C31588; LifeSpan Biosciences, Seattle, WA, USA) diluted at $1.5 \mu \mathrm{g} / \mathrm{ml}$, a goat polyclonal antibody for Wnt5B (C-16) diluted at 1:100, a mouse monoclonal antibody for survivin (sc17779) diluted at 1:50, a mouse monoclonal antibody for c-Myc (9E10) (all from Santa Cruz Biotechnology, Inc.) diluted at 1:100, and a mouse monoclonal antibody for the Ki-67 antigen (MIB-1; Dako, Glostrup, Denmark) diluted at 1:40. Formalin-fixed paraffin-embedded tissue was cut into $4-\mu \mathrm{m}$ sections and mounted on poly-lysine-coated slides. After deparaffinization and rehydration, the slides were heated in a microwave for $10 \mathrm{~min}$ in $10 \mu \mathrm{mol} / \mathrm{l}$ citrate buffer solution at $\mathrm{pH}$ 6.0. After quenching the endogenous peroxidase activity with $0.3 \%$ $\mathrm{H}_{2} \mathrm{O}_{2}$ (in absolute methanol) for $30 \mathrm{~min}$, the sections were treated with $5 \%$ bovine serum albumin. Duplicate sections were incubated overnight with the primary antibodies, respectively. Slides were then incubated for $1 \mathrm{~h}$ with biotinylated secondary antibodies (Vector Laboratories, Burlingame, CA, USA). The sections were incubated with the avidin-biotinperoxidase complex (Vector) for $1 \mathrm{~h}$, and antibody binding was visualized with 3,3'-diaminobenzidine tetrahydrochloride. Lastly, the sections were lightly counterstained with Mayer's hematoxylin.

The immunostained sections were examined by two authors (M.K. and C.H.) without knowledge of the patient characteristics. Cases with discrepancies were jointly reevaluated until a consensus was reached. At least 200 cells were scored per x40 field about tumour cells. The percentage of carcinoma cells with positive staining for Ki-67 in a given specimen was scored as the Ki-67 proliferation index (9).

Detection of apoptosis. The TUNEL method was performed using the In Situ Apoptosis Detection kit (Takara Biomedicals, Otsu, Japan). After deparaffinization and rehydration, the sections were treated with $20 \mu \mathrm{g} / \mathrm{ml}$ proteinase $\mathrm{K}$ for $15 \mathrm{~min}$. After quenching the endogenous peroxidase activity with $3 \%$ $\mathrm{H}_{2} \mathrm{O}_{2}$ for $5 \mathrm{~min}$, the sections were incubated for $90 \mathrm{~min}$ at $37^{\circ} \mathrm{C}$ with the TUNEL reaction mixture, including terminal deoxynucleotidyl transferase (TdT). Next, the sections were incubated for $30 \mathrm{~min}$ at $37^{\circ} \mathrm{C}$ with anti-FITC horseradish peroxidase conjugate. Staining was detected by 3,3'-diaminobenzidine tetrahydrochloride incubation for $15 \mathrm{~min}$. Lastly, the sections were lightly counterstained with Mayer's hematoxylin. In each case, a total of 10,000 tumor cells were evaluated by two authors (M.K. and C.H.) independently, without knowledge of the patient characteristics. The apoptotic index was defined as the number of apoptotic cells per 1,000 tumor cells (18).

Statistical analysis. The statistical significance of Wnt1, Wnt2B, Wnt5A, survivin, and c-Myc expression was assessed by t-test, ANOVA with Bonferroni/Dunn test or Pearson's correlation coefficient. The Kaplan-Meier method was used to estimate the probability of overall survival as a function of time, and differences in the survival of subgroups of patients were compared by using Mantel's log-rank test. A multivariate analysis was performed using the Cox regression model to study the effects of different variables on survival. The sample was classified as a Wnt1-high tumor when the percentage of Wnt1-positive tumor cells was $>50 \%$, and as a Wnt5A-high tumor when the percentage of Wnt5A-positive tumor cells was $>30 \%$, as reported previously $(8,9)$. The sample was classified as a Wnt2B-high tumor when the percentage of Wnt2Bpositive tumor cells was $>50 \%$, a nuclear survivin-high tumor when the percentage of nuclear survivin-positive tumor cells was $>20 \%$, and a c-Myc-high tumor when the percentage of c-Myc-positive tumor cells was $>30 \%$, as this had the highest significance value in relation to the $\mathrm{Ki}-67$ proliferation index. The sample was classified as a $\mathrm{Ki}-67$-high tumor when the Ki-67 proliferation index was $>30 \%$ as this had the highest significance value in relation to patient survival. All p-values were based on two-tailed statistical analysis, and a p-value of $<0.05$ was considered to indicate statistical significance.

\section{Results}

Wntl expression in MPMs. The Wnt1 expression appeared in a cytoplasmic staining pattern (Fig. 1A). The percentage of Wnt1-positive tumor cells varied greatly among the MPM

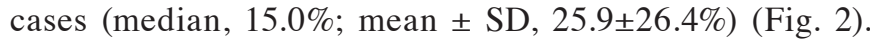
Regarding clinical and pathological characteristics, the percentage of Wnt1-positive tumors was significantly higher in stage I patients $(\mathrm{p}=0.0107)$ (Table I). However, no significant difference was observed in the Wnt1 status according to tumor histology.

Wnt $2 B$ expression in MPMs. The Wnt2B expression was also detected in a cytoplasmic staining pattern (Fig. 1B). The percentage of Wnt2B-positive tumor cells also varied greatly among the MPM cases (median, 60.0\%; mean \pm SD, $54.1 \pm 31.7 \%$ ) (Fig. 2). No significant difference was observed in the Wnt2B status according to pathological stage and tumor histology (Table I).

Wnt5A expression in MPMs. The Wnt5A expression appeared in a cytoplasmic staining pattern (Fig. $1 \mathrm{H})$. The percentage of Wnt5A-positive tumor cells varied greatly among the MPM cases (median, 30.0\%; mean $\pm \mathrm{SD}, 32.1 \pm 27.9 \%$ ) (Fig. 2). The percentage of Wnt5A-positive tumors was significantly higher in stage I patients $(\mathrm{p}=0.0466)$ (Table I). However, no significant difference was observed in the Wnt5A status according to tumor histology (Table I).

Predominant expression of different Wht proteins in MPMs. Among the 107 MPMs, 23 MPMs (21.5\%) were Wnt1-high tumors, 72 MPMs (67.3\%) were Wnt2B-high tumors, and 54 MPMs (50.5\%) were Wnt5A-high tumors. There was no correlation between the expression levels of the different Wnt proteins. Furthermore, the percentage of Wnt2B-positive tumors was significantly higher than that of the other Wnt members $(\mathrm{p}<0.0001$ vs. Wnt1 and $\mathrm{p}<0.0001$ vs. Wnt5A) (Fig. 2).

Survivin expression in MPMs. Immunostaining of the antibody against survivin showed various patterns of nuclear staining 

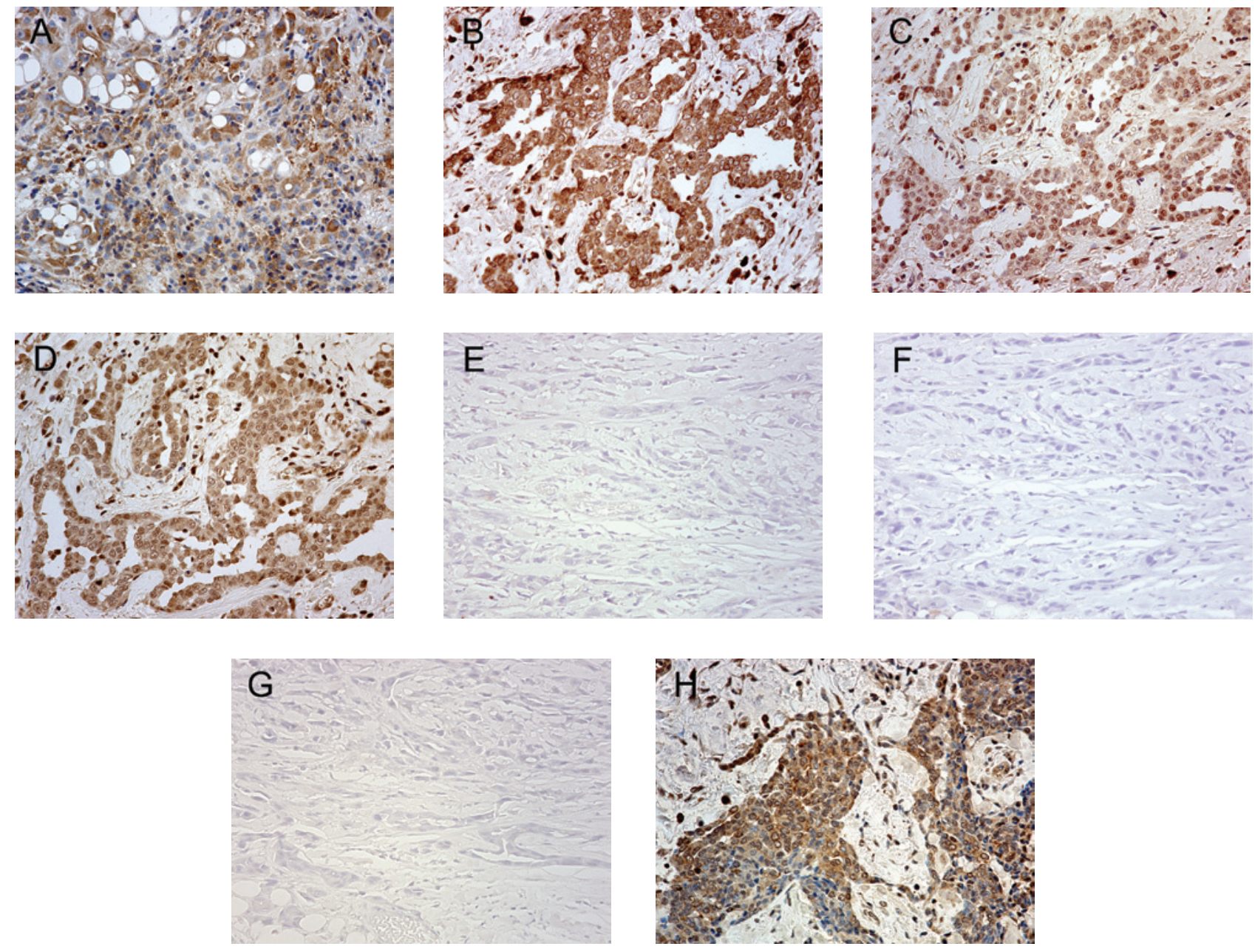

Figure 1. Immunohistochemical staining of human MPM tissue using the avidin-biotin-peroxidase complex procedure. (A) A Wnt1-high tumor. A tumor with positive expression of (B) Wnt2B, (C) nuclear survivin and (D) c-Myc. A tumor with negative expression of (E) Wnt2B, (F) nuclear survivin and (G) c-Myc. (H) A Wnt5A-high tumor. Original magnification, x200.

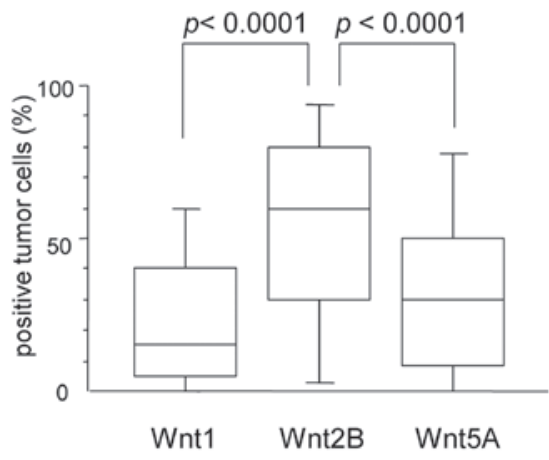

Figure 2. Distribution of Wnt1, Wnt2B and Wnt5A expression in 107 MPMs.

and cytoplasmic staining (Fig. 1C). The percentage of nuclear survivin staining was significantly higher than that of cytoplasmic survivin staining $(27.2 \pm 30.2$ vs. $15.6 \pm 25.6 \%, \mathrm{p}=0.0025)$.

Regarding tumor biology, the Ki-67 proliferation index was significantly higher in nuclear survivin-high tumors than in nuclear survivin-low tumors $(60.6 \pm 33.8$ vs. $37.8 \pm 27.8 \%$, $\mathrm{p}=0.0002)($ Fig. 3A). In contrast, there was no difference in the apoptotic index according to the cytoplasmic survivin expres- sion in MPMs (7.0 \pm 3.7 in cytoplasmic survivin-high tumors and $5.7 \pm 2.3$ in cytoplasmic survivin-low tumors).

Regarding the Wnt status, the percentage of survivin-positive tumor cells significantly correlated with the percentage of Wnt2B-positive tumor cells $(r=0.335, p<0.001)$. However, the percentage of survivin-positive tumor cells did not correlated with the percentage of Wnt1-positive tumor cells $(\mathrm{p}=0.1101)$ or the percentage of Wnt5A-positive tumor cells $(\mathrm{p}=0.4631)$.

c-Myc expression in MPMs. The percentage of c-Myc-positive tumor cells varied greatly among the MPM cases (median, $35.0 \%$; mean \pm SD, 37.3 $\pm 30.3 \%$ ) (Fig. 1D). Regarding tumor proliferation, the Ki-67 proliferation index was significantly higher in c-Myc-high tumors than in c-Myc-low tumors $(53.8 \pm 31.4$ vs. $39.7 \pm 31.7 \%$; $p=0.0231)$ (Fig. $3 \mathrm{~B})$. In contrast, there was no difference in the apoptotic index in relation to the c-Myc status in MPMs (5.2 \pm 2.5 in c-Myc-high tumors and $7.1 \pm 3.0$ in c-Myc-low tumors).

In regards to the Wnt status, the percentage of c-Myc-positive tumor cells significantly correlated with the percentage of Wnt2B-positive tumor cells $(r=0.364, p<0.001)$. However, the percentage of c-Myc-positive tumor cells did not correlated with the percentage of Wnt1-positive tumor cells $(p=0.3937)$ or the percentage of Wnt5A-positive tumor cells $(\mathrm{p}=0.3498)$. 
Table I. Expression of Wnt family members in 107 patients with malignant pleural mesothelioma according to clinicopathological characteristics.

\begin{tabular}{|c|c|c|c|c|c|c|}
\hline & \multicolumn{2}{|c|}{ Wnt1 } & \multicolumn{2}{|c|}{ Wnt2B } & \multicolumn{2}{|c|}{ Wnt5A } \\
\hline & $\begin{array}{c}\text { Positive } \\
\text { tumors (\%) }\end{array}$ & P-value & $\begin{array}{c}\text { Positive } \\
\text { tumors }(\%)\end{array}$ & P-value & $\begin{array}{c}\text { Positive } \\
\text { tumors (\%) }\end{array}$ & P-value \\
\hline \multicolumn{7}{|l|}{ Age (years) } \\
\hline$<65$ & $25.5 \pm 28.5$ & 0.8504 & $55.7 \pm 33.8$ & 0.6150 & $34.6 \pm 29.8$ & 0.3764 \\
\hline$\geq 65$ & $26.4 \pm 24.4$ & & $52.6 \pm 29.7$ & & $29.8 \pm 25.8$ & \\
\hline \multicolumn{7}{|l|}{ Gender } \\
\hline Male & $24.3 \pm 25.2$ & 0.2947 & $54.3 \pm 31.4$ & 0.9470 & $32.4 \pm 28.1$ & 0.8772 \\
\hline Female & $30.5 \pm 29.9$ & & $53.8 \pm 33.2$ & & $31.5 \pm 27.6$ & \\
\hline \multicolumn{7}{|l|}{ Asbestos exposure } \\
\hline Yes & $19.7 \pm 22.6$ & 0.0588 & $52.5 \pm 30.2$ & 0.2384 & $28.3 \pm 27.4$ & 0.1411 \\
\hline No & $30.3 \pm 27.0$ & & $60.5 \pm 29.6$ & & $37.3 \pm 26.0$ & \\
\hline \multicolumn{7}{|l|}{ Smoking } \\
\hline Non-smoker & $25.1 \pm 26.3$ & 0.7582 & $55.7 \pm 30.6$ & 0.6461 & $33.6 \pm 26.3$ & 0.6194 \\
\hline Smoker & $26.7 \pm 26.7$ & & $52.8 \pm 32.8$ & & $30.9 \pm 29.3$ & \\
\hline \multicolumn{7}{|l|}{ Pathological stage } \\
\hline I & $44.6 \pm 32.3$ & 0.0107 & $67.4 \pm 29.2$ & 0.2180 & $46.5 \pm 27.1$ & 0.0466 \\
\hline II & $29.8 \pm 30.0$ & & $56.7 \pm 27.6$ & & $38.3 \pm 29.7$ & \\
\hline III-IV & $21.6 \pm 22.9$ & & $51.1 \pm 32.9$ & & $28.0 \pm 26.7$ & \\
\hline \multicolumn{7}{|l|}{ Histology } \\
\hline Epithelioid & $29.2 \pm 28.5$ & 0.2649 & $58.6 \pm 31.0$ & 0.1654 & $34.9 \pm 29.0$ & 0.4713 \\
\hline Sarcomatoid & $19.1 \pm 19.8$ & & $47.0 \pm 30.1$ & & $32.9 \pm 25.5$ & \\
\hline Biphasic & $27.5 \pm 26.3$ & & $54.9 \pm 32.8$ & & $23.4 \pm 23.4$ & \\
\hline Desmoplastic & $13.1 \pm 21.5$ & & $33.9 \pm 34.9$ & & $32.1 \pm 36.3$ & \\
\hline Total number of patients & $25.9 \pm 26.4$ & & $54.1 \pm 31.7$ & & $32.1 \pm 27.9$ & \\
\hline
\end{tabular}
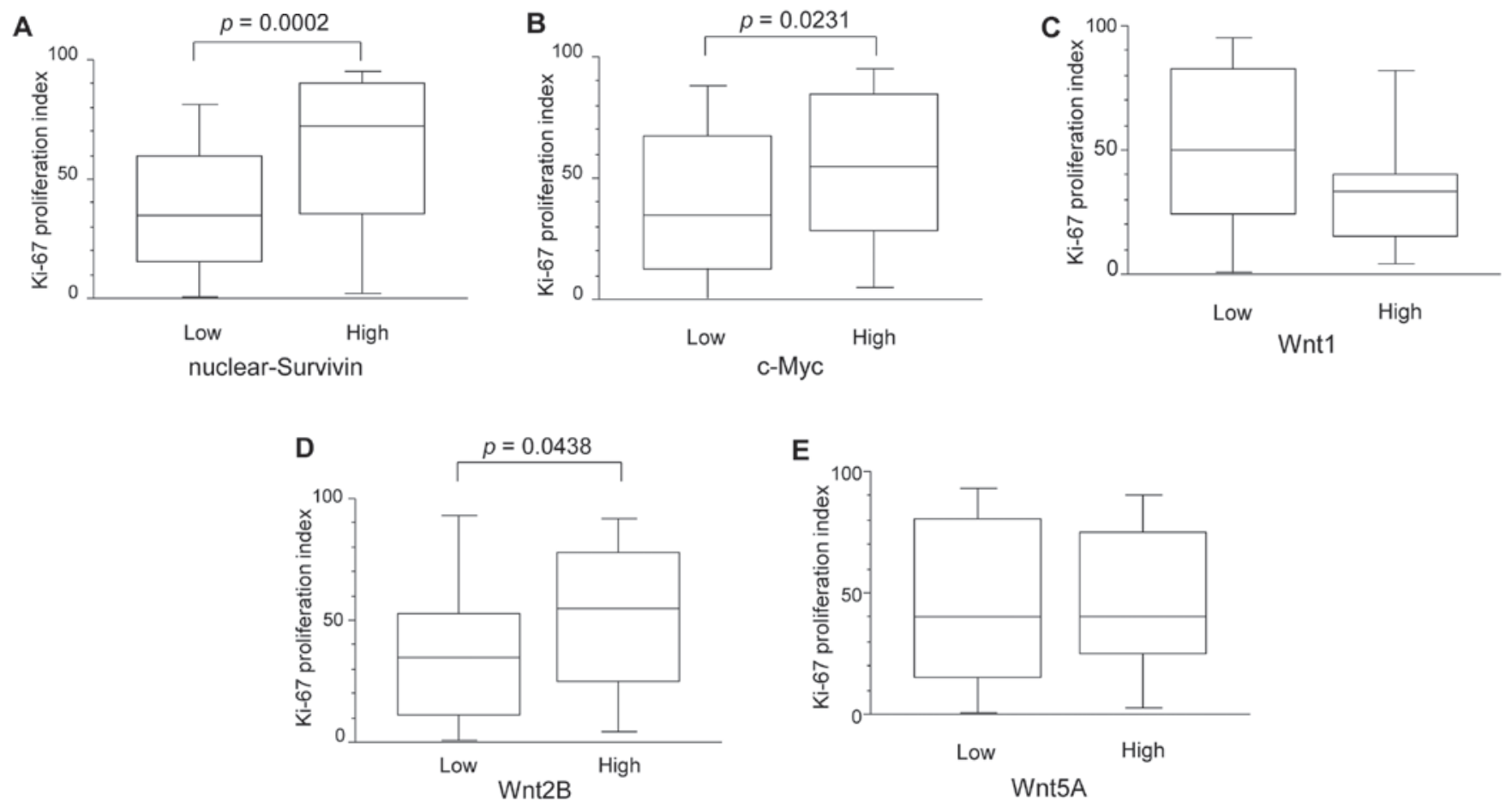

Figure 3. Ki-67 proliferation index of MPMs in relation to (A) nuclear survivin, (B) c-Myc, (C) Wnt1, (D) Wnt2B and (E) Wnt5A status. 

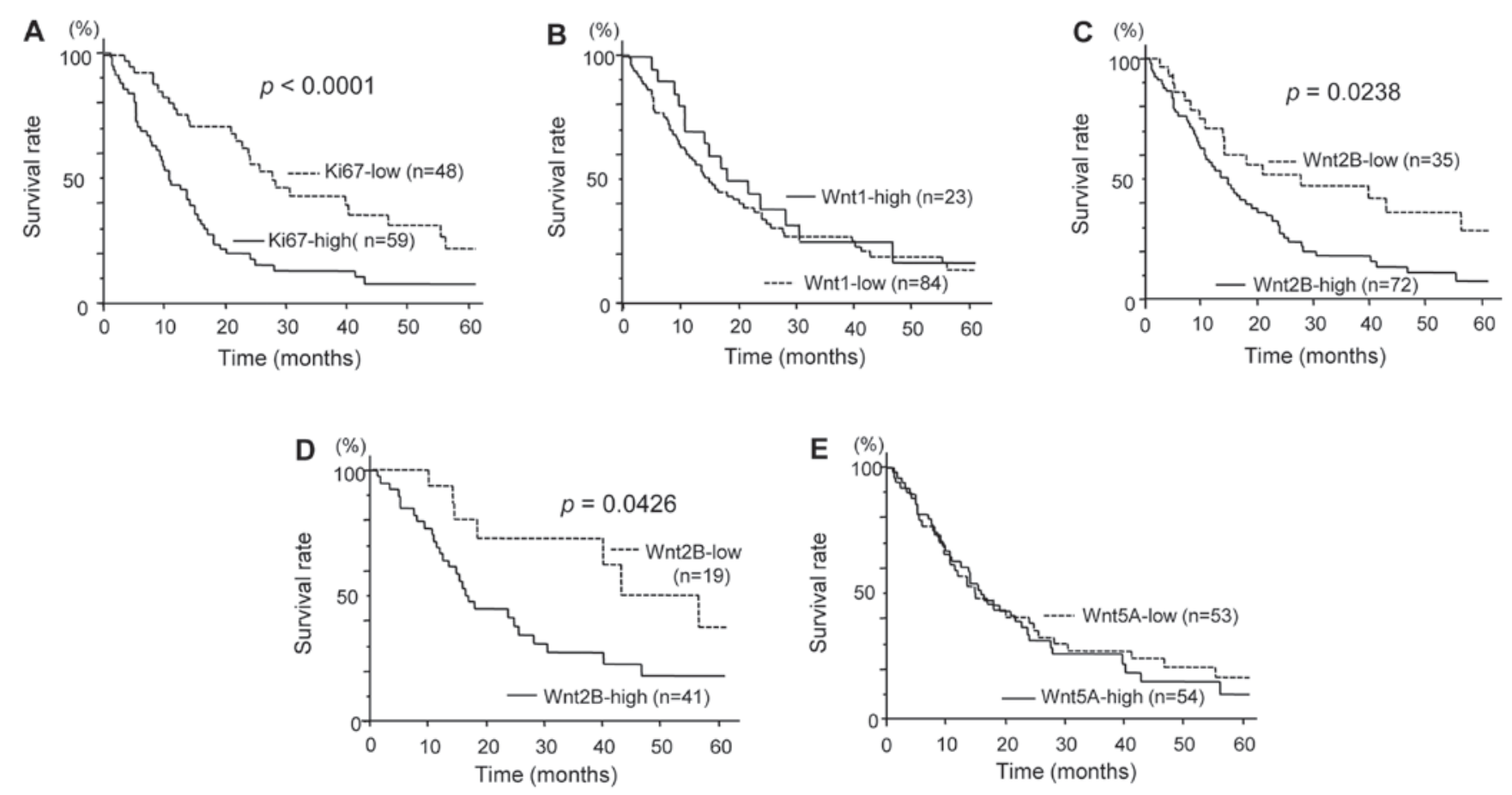

Figure 4. (A) Overall survival of 107 MPM patients in relation to Ki-67 proliferation index, (B) overall survival of 107 MPM patients in relation to Wnt1 status, (C) overall survival of 107 MPM patients in relation to Wnt2B status, (D) overall survival of 60 patients with epithelioid tumors in relation to Wnt2B status, (E) overall survival of 107 MPM patients in relation to Wnt5A status.

Tumor biology and intratumoral Wnt1 status in MPMs. There was no difference in the Ki-67 proliferation index in relation to the Wnt1 status in MPMs (35.4 $\pm 28.2 \%$ in Wnt1-high tumors and $50.1 \pm 32.7 \%$ in Wnt1-low tumors) (Fig. 3C). Furthermore, there was no difference in the apoptotic index in relation to the Wnt1 status in MPMs (11.2 \pm 6.0 in Wnt1-high tumors and $4.7 \pm 1.8$ in Wnt1-low tumors).

Tumor biology and intratumoral Wnt2B status in MPMs. Regarding tumor proliferation, the Ki-67 proliferation index was $51.3 \pm 31.4 \%$ in Wnt2B-high tumors, and $37.9 \pm 32.4 \%$ in Wnt2B-low tumors (Fig. 3D). The Ki-67 proliferation index was significantly higher in Wnt2B-high tumors than in Wnt2B-low tumors ( $\mathrm{p}=0.0438$ ). In contrast, there was no difference in the apoptotic index in relation to the Wnt $2 \mathrm{~B}$ status in MPMs (7.7 \pm 2.8 in Wnt2B-high tumors and 2.9 \pm 1.3 in Wnt2B-low tumors).

Tumor biology and intratumoral Wnt5A status in MPMs. There was no difference in the Ki-67 proliferation index in relation to the Wnt5A status in MPMs $(48.0 \pm 31.1 \%$ in Wnt5Ahigh tumors and $45.9 \pm 33.6 \%$ in Wnt5A-low tumors) (Fig. 3E). In addition, there was no difference in the apoptotic index in relation to the Wnt 5A status in MPMs (5.6 2 2.6 in Wnt5A-high tumors and $6.6 \pm 2.9$ in Wnt5A-low tumors).

Survival of MPM patients in relation to the Ki-67 proliferation index and intratumoral Wnt status. Regarding tumor proliferation, the 5-year survival rate was significantly lower in patients with high Ki-67 tumors than in those with low Ki-67 tumors (7.8 vs. 30.4\%, p<0.0001) (Fig. 4A). Regarding the intratumoral
Wnt status, the 5-year survival rate was $11.6 \%$ in patients with Wnt2B-high tumors, and 32.3\% in patients with Wnt2B-low tumors (Fig. 4C). The overall survival was significantly lower in patients with Wnt2B-high tumors than in those with Wnt2B-low tumors $(p=0.0238)$. In particular, the overall survival was significantly lower in patients with Wnt2B-high epithelioid tumors than in those with Wnt2B-low epithelioid tumors (20.9 vs. $37.4 \%$ at 5 -year survival, $p=0.0426$ ) (Fig. $4 \mathrm{D}$ ). In contrast, there was no difference in patient survival according to Wnt1 status or Wnt5A status (Fig. 4B and E). A Cox multivariate analysis demonstrated that Wnt2B status (hazard ratio 2.396; $\mathrm{p}=0.0042$ ), pathological stage (hazard ratio $1.455 ; \mathrm{p}=0.0439$ ), and tumor histology (hazard ratio 1.973; $\mathrm{p}=0.0074$ ) were significant prognostic factors for MPM patients (Table II).

\section{Discussion}

The clinical outcome of MPM patients is poor even in patients with early-stage MPM (3). In fact, $69.2 \%$ (74 of 107) of MPM patients included in the present study had advanced-stage MPM, and the 5-year survival rate was only $22.2 \%$ even in stage I patients. The development of new treatment strategies for MPM patients is therefore critical.

The Wnt family is involved in the regulation of a wide variety of normal and pathological processes including tumorigenesis $(5,6)$. We first investigated the expression of Wnt1, Wnt2B, and Wnt5A in tumor tissues from MPM patients. The present study showed that the intratumoral expression of Wnt2B was significantly higher than that of Wnt1 and Wnt5A in MPMs. In addition, Wnt1 and Wnt5A expression levels were significantly lower in MPMs than in non-small cell lung cancers that were 
Table II. Multivariate regression analysis for predicting survival of 107 patients with malignant pleural mesothelioma.

\begin{tabular}{|c|c|c|c|c|}
\hline Variables & $\begin{array}{l}\text { Assigned } \\
\text { score }\end{array}$ & $\begin{array}{c}\text { Hazard } \\
\text { ratio }\end{array}$ & $95 \% \mathrm{CI}$ & P-value \\
\hline \multicolumn{5}{|l|}{ Wnt2B status } \\
\hline Low & 0 & 2.396 & $1.317-4.359$ & 0.0042 \\
\hline High & 1 & & & \\
\hline \multicolumn{5}{|l|}{ Age (years) } \\
\hline$<65$ & 0 & 1.114 & $0.664-1.871$ & 0.6821 \\
\hline$\geq 65$ & 1 & & & \\
\hline \multicolumn{5}{|l|}{ Gender } \\
\hline Male & 0 & 1.434 & $0.772-2.665$ & 0.2536 \\
\hline Female & 1 & & & \\
\hline \multicolumn{5}{|c|}{ Asbestos exposure } \\
\hline No & 0 & 0.745 & $0.441-1.259$ & 0.2710 \\
\hline Yes & 1 & & & \\
\hline \multicolumn{5}{|l|}{ Smoking } \\
\hline Non-smoker & 0 & 0.989 & $0.577-1.696$ & 0.9692 \\
\hline Smoker & 1 & & & \\
\hline \multicolumn{5}{|l|}{ Clinical stage } \\
\hline $\mathrm{I}$ & 0 & 1.455 & $1.010-2.095$ & 0.0439 \\
\hline II & 1 & & & \\
\hline III-IV & 2 & & & \\
\hline \multicolumn{5}{|l|}{ Histology } \\
\hline Epithelioid & 0 & 1.973 & $1.200-3.245$ & 0.0074 \\
\hline Others & 1 & & & \\
\hline
\end{tabular}

CI, confidence interval.

analyzed concurrently (data not shown). These results suggest that the intratumoral Wnt2B expression has an effect on the tumor biology of MPMs.

Wnt2B is known to stimulate the canonical Wnt/ $\beta$-catenin pathway (19). The activation of the canonical Wnt/ $\beta$-catenin pathway leads to the transcription of Wnt-target genes including survivin $(13,14)$, c-Myc $(15)$, and vascular endothelial growth factor-A (20). Therefore, Wnt2B overexpression may affect tumor biology during tumor progression through the induction of these tumor-associated Wnt targets.

Furthermore, the present study clearly demonstrated that the tumor proliferation rate was associated with survival of MPM patients. In fact, MPM is clinically characterized by rapid and diffuse local growth, which results in a poor prognosis. In contrast, the apoptotic index in MPM tissues was significantly lower than that in non-small cell lung cancers that were analyzed concurrently (data not shown). We, therefore, evaluated the expression of survivin $(13,14)$ and c-Myc $(15)$, Wnt-targets associated with proliferation.

Previous studies have shown that survivin not only inhibits the caspase-dependent apoptotic pathway $(21,22)$, but also accelerates cell proliferation (23). In particular, the nuclear localization of survivin affects cell mitosis through chromosome condensation and segregation $(24,25)$. Previous studies revealed that the nuclear expression of survivin is associated with tumor proliferation and a poor prognosis in cancer patients
(26-28). The present study also demonstrated that the nuclear expression of survivin was associated with tumor proliferation in MPMs. Furthermore, the survival was significantly lower in patients with nuclear survivin-high tumors than in those with nuclear survivin-low tumors $(\mathrm{p}=0.0447)$ (data not shown).

$\mathrm{c}-\mathrm{Myc}$ is also a target of the canonical Wnt $/ \beta$-catenin pathway (15). c-Myc is involved in cell cycle progression through the stimulation and repression of the expression of cell cycle regulators (29). Previous studies have revealed that c-Myc overexpression is associated with the malignant phenotype in various human cancers $(8,30)$. The present clinical study also demonstrated that c-Myc expression was associated with the tumor proliferation of MPMs.

Finally, we investigated the clinical significance of Wnt expression in relation to survivin and c-Myc expression. Consequently, the present study has revealed that the intratumoral Wnt2B expression is associated with survivin and c-Myc expression, which results in the acceleration of tumor proliferation. Furthermore, overall survival was lower in patients with Wnt2B-high tumors than in those with Wnt2B-low tumors. To our knowledge, this is the first comprehensive clinical study clearly demonstrating the clinical significance of intratumoral Wnt2B expression in MPMs. In conclusion, the present study demonstrated that intratumoral Wnt2B expression is associated with tumor proliferation and survival of MPM patients through the induction of survivin 
and c-Myc. Wnt2B is therefore a potential candidate for molecular-targeted therapy for MPMs. In fact, it was recently demonstrated that an adenoviral vector expressing short hairpin RNA (shRNA) against Wnt2B had a strong antitumor effect against Wnt2B-overexpressing tumors, via the downregulation of survivin and $\mathrm{c}-\mathrm{Myc}$, resulting in the inhibition of tumor proliferation and the induction of apoptosis (31). Therefore, Wnt2B-inhibiting gene therapy, including the intrathoracic administration of viral vectors (32) or non-viral vectors (33), may be effective as a therapeutic strategy for Wnt2B-overexpressing MPMs (34).

\section{Acknowledgements}

We thank Ms. Seiko Sakai for the excellent secretarial assistance.

\section{References}

1. Greillier L and Astoul P: Mesothelioma and asbestos-related pleural diseases. Respiration 76: 1-15, 2008.

2. Bueno R: Mesothelioma clinical presentation. Chest 116 (Suppl 6): S444-S445, 1999.

3. Fujimoto N, Aoe K, Gamba K, Kato K, Yamazaki K and Kishimoto T: Clinical investigation of malignant mesothelioma in Japan. J Cancer Res Clin Oncol 136: 1755-1759, 2010.

4. Schil PE, Bass P, Gaafar R, et al: Trimodality therapy for malignant pleural mesothelioma: results from an EORTC phase II multicentre trial. Eur Respir J 36: 1362-1369, 2010.

5. Dale TC: Signal transduction by the Wnt family of ligands. Biochem J 329: 209-223, 1998.

6. You Z, Saims D, Chen S, et al: Wnt signaling promotes oncogenic transformation by inhibiting c-Myc-induced apoptosis. J Cell Biol 157: 429-440, 2002.

7. Huang C, Liu D, Masuya D, et al: MRP-1/CD9 gene transduction downregulates Wnt signal pathways. Oncogene 23: 7475-7483, 2004.

8. Huang C, Liu D, Ishikawa S, et al: Wnt1 overexpression promotes tumour progression in non-small cell lung cancer. Eur J Cancer 44: 2680-2688, 2008

9. Huang C, Liu D, Nakano J, et al: Wnt5a expression is associated with the tumor proliferation and the stromal vascular endothelial growth factor-A expression in non-small cell lung cancer. J Clin Oncol 23: 8765-8773, 2005.

10. Chen G, Shukeir N, Potti A, et al: Up-regulation of Wnt-1 and $\beta$-catenin production in patients with advanced metastatic prostate carcinoma: potential pathogenetic and prognostic implications. Cancer 101: 1345-1356, 2004.

11. Zhang WM, Lo Muzio L, Rubini C and Yan G: Effect of WNT-1 on $\beta$-catenin expression and its relation to $\mathrm{Ki}-67$ and tumor differentiation in oral squamous cell carcinoma. Oncol Rep 13: 1095-1099, 2005.

12. Lee A, Raz DJ, He B and Jablons DM: Update on the molecular biology of malignant mesothelioma. Cancer 109: 1454-1461, 2007.

13. Kim PJ, Plescia J, Clevers H, Fearon ER and Altieri DC: Survivin and molecular pathogenesis of colorectal cancer. Lancet 362: 205-209, 2003

14. Ma H, Nguyen C, Lee KS and Kahn M: Differential roles for the coactivators $\mathrm{CBP}$ and $\mathrm{p} 300$ on $\mathrm{TCF} / \beta$-catenin-mediated survivin gene expression. Oncogene 24: 3619-3631, 2005.
15. He TC, Sparks AB, Rago C, et al: Identification of c-MYC as a target of the APC pathway. Science 281: 1509-1512, 1998.

16. Husain AN, Colby TV, Ordonez NG, et al: Guidelines for pathologic diagnosis of malignant mesothelioma. Arch Pathol Lab Med 133: 1317-1331, 2009.

17. Attanoos RL and Gibbs AR: Pathology of malignant mesothelioma. Histopathology 30: 403-418, 1997.

18. Nakano J, Huang C, Liu D, et al: The clinical significance of splice variants and subcellular localization of survivin in non-small cell lung cancer. Br J Cancer 98: 1109-1117, 2008.

19. Katoh M, Kirikoshi H, Terasaki H and Shiokawa K: WNT2B2 mRNA, up-regulated in primary gastric cancer, is a positive regulator of the WNT-beta-catenin-TCF signaling pathway. Biochem Biophys Res Commun 289: 1093-1098, 2001.

20. Zhang X, Gaspard JP and Chung DC: Regulation of vascular endothelial growth factor by the Wnt and K-ras pathways in colonic neoplasia. Cancer Res 61: 6050-6054, 2001.

21. Lu CD, Altieri DC and Tanigawa N: Expression of a novel antiapoptosis gene, survivin, correlated with tumor cell apoptosis and p53 accumulation in gastric carcinomas. Cancer Res 58: 1808-1812, 1998.

22. Kawasaki H, Altieri DC, Lu CD, Toyoda M, Tenjo T and Tanigawa N: Inhibition of apoptosis by survivin predicts shorter survival rates in colorectal cancer. Cancer Res 58: 5071-5074, 1998.

23. Mita AC, Mita MM, Nawrocki ST and Giles FJ: Survivin: key regulator of mitosis and apoptosis and novel target for cancer therapeutics. Clin Cancer Res 14: 5000-5005, 2008.

24. Li F, Ambrosini G, Chu EY, et al: Control of apoptosis and mitotic spindle checkpoint by survivin. Nature 396: 580-584, 1998.

25. Adams RR, Carmena M and Earnshaw WC: Chromosomal passengers and the (aurora) ABCs of mitosis. Trends Cell Biol 11: 49-54, 2001.

26. Martinez A, Bellosillo B, Bosch F, et al: Nuclear survivin expression in mantle cell lymphoma is associated with cell proliferation and survival. Am J Pathol 164: 501-510, 2004.

27. Shinohara ET, Gonzalez A, Massion PP, et al: Nuclear survivin predicts recurrence and poor survival in patients with resected nonsmall cell lung carcinoma. Cancer 103: 1685-1692, 2005.

28. Brennan DJ, Rexhepaj E, O'Brien SL, et al: Altered cytoplasmicto nuclear ratio of survivin is a prognostic indicator in breast cancer. Clin Cancer Res 14: 2681-2689, 2008.

29. Dang CV, Resar LM, Emison E, et al: Function of the c-Myc oncogenic transcription factor. Exp Cell Res 253: 63-77, 1999.

30. Yang G, Timme TL, Frolov A, Wheeler TM and Thompson TC: Combined c-Myc and caveolin-1 expression in human prostate carcinoma predicts prostate carcinoma progression. Cancer 103: 1186-1194, 2005

31. Lu D, Kadota K, Ueno M, Nakashima N, Yokomise H and Huang CL: Adenoviral vector expressing short hairpin RNA targeting Wnt2B has an effective antitumour activity against Wnt2B2-overexpressing tumours. Eur J Cancer: Jun 4, 2011 (Epub ahead of print).

32. Rao DD, Vorhies JS, Senzer N, et al: siRNA vs. shRNA: similarities and differences. Adv Drug Deliv Rev 61: 746-759, 2009.

33. Ma B, Zhang S, Jiang H, Zhao B and Lv H: Lipoplex morphologies and their influences on transfection efficiency in gene delivery. J Control Release 123: 184-194, 2007.

34. Watanabe Y, Kojima T, Kagawa S, et al: A novel translational approach for human malignant pleural mesothelioma: heparanase-assisted dual virotherapy. Oncogene 29: 1145-1154, 2010. 